\title{
A 3D coupled hydro-mechanical granular model for the prediction of hot tearing formation
}

\author{
M. Sistaninia ${ }^{1}$, A.B. Phillion ${ }^{2}$, J.-M. Drezet ${ }^{1}$, M. Rappaz ${ }^{1}$ \\ 1. Computational Materials Laboratory, Ecole Polytechnique Fédérale de Lausanne, \\ Lausanne, Switzerland \\ 2. Okanagan School of Engineering, University of British Columbia, Kelowna, Canada \\ E-mail: meisam.sistaninia@epfl.ch
}

\begin{abstract}
A new 3D coupled hydro-mechanical granular model that simulates hot tearing formation in metallic alloys is presented. The hydro-mechanical model consists of four separate 3D modules. (I) The Solidification Module (SM) is used for generating the initial solidliquid geometry. Based on a Voronoi tessellation of randomly distributed nucleation centers, this module computes solidification within each polyhedron using a finite element based solute diffusion calculation for each element within the tessellation. (II) The Fluid Flow Module (FFM) calculates the solidification shrinkage and deformation-induced pressure drop within the intergranular liquid. (III) The Semi-solid Deformation Module (SDM) is used to simulate deformation of the granular structure via a combined finite element / discrete element method. In this module, deformation of the solid grains is modeled using an elasto-viscoplastic constitutive law. (IV) The Failure Module (FM) is used to simulate crack initiation and propagation with the fracture criterion estimated from the overpressure required to overcome the capillary forces at the liquid-gas interface. The FFM, SDM, and FM are coupled processes since solid deformation, intergranular flow, and crack initiation are deeply linked together. The granular model predictions have been validated against bulk data measured experimentally and calculated with averaging techniques.
\end{abstract}

\section{Introduction}

Hot tearing is a spontaneous failure of the semi-solid state that occurs in many metallic alloys and which results in an intergranular fracture profile. The thermal strains induced during casting due to both solidification shrinkage and thermal contraction of the solid skeleton can lead to the formation of hot tears. In order for simulations to successfully predict the formation of this defect during solidification, both the interaction between the solid and liquid phases as well as the evolution in microstructure that occurs during solidification must be included. It has recently been demonstrated that partially solidified alloys can exhibit the characteristics of a cohesionless granular material, including Reynolds' dilatancy, arching, jamming and stick-slip flow [1-3]. In this regard, the semi-solid behavior is quite complex in comparison with fluid flow and solid deformation. Fluid flow involves large deformations and low stresses, whereas solid deformation involves the opposite, i.e., high stresses and small deformations. Not surprisingly, modeling the semi-solid regime remains a challenging task. Most recent modeling studies of hot tearing are based on macro scale averaging methods for modeling the interaction between a deforming solid skeleton and intergranular fluid flow (e.g. [4-7]). The main weakness of such approaches is that they cannot account for the localization of straining and feeding at grain boundaries, a feature that has been clearly evidenced recently by in-situ X-ray tomography tensile tests [3]. Recently, 
a number of authors have begun to consider the semi-solid as a granular material to describe equiaxed-globular solidification [8-10], mechanical behavior [11-14] and liquid feeding [15-18]. Although most of the granular semi-solid deformation models were successful in modeling the mechanical behavior of semi-solid alloys before fracture initiation, they were not successful in modeling the failure stage, i.e., hot tear formation. In order to reach such a goal, the formation and percolation of voids within the intergranular liquid channels need to be considered, a feature that has not been addressed in previous models. On the other hand, previous fluid flow models [15-18] made the major assumption that the grains remain fixed. In the present study, a 3D coupled hydro-mechanical granular model has been developed to overcome the limitations.

\section{Model development}

The granular model is made of four separate 3D modules: (I) A Solidification Module (SM) for generating the initial solid-liquid structure [9]; (II) A Fluid Flow Module (FFM) for pressure drop calculation [18]; (III) A Semi-solid Deformation Module (SDM) for localization of straining [14]; and (IV) A Failure Module (FM) for modeling crack initiation and propagation. The FFM, SDM, and FM are coupled processes since solid deformation, intergranular flow, and crack initiation and propagation are deeply linked together and a fully hydro-mechanical coupling is used between the FFM and SDM. In the present work, the material simulated is an Al$2 \mathrm{wt} . \% \mathrm{Cu}$ alloy, due to its long freezing range, propensity for hot tear formation, and availability of experimental constitutive data (e.g. $[7,19,20])$.

\subsection{Solidification Module}

The 3D granular model of solidification known as GMS-3D [9] is an extension of the previous work by Mathier et al. [10] and Vernède et al. [21]. In this simulation, the grain geometry is derived from a Voronoi tessellation of random nucleation centres with each Voronoi region assumed to be a single grain. Each polyhedral grain derived from the Voronoi tessellation (Fig. 1 ) is subdivided into a set of pyramids having the nucleation center as the summit and the Voronoi facet as the base. The base of the pyramid is subdivided into triangles so as to only have tetrahedral elements that can be used either in SDM or FFM calculations (see Fig. 1(b)). Because of their regular shape, solidification within a tetrahedron can be reduced to a onedimensional problem that is calculated using a micro-segregation model with infinite mixing in the liquid and back-diffusion in the solid. While all tetrahedra within a pyramid solidify at the same speed, since they have the same perpendicular length and hence the same Fourier number in the 1D solidification simulation, elements from different pyramids solidify at different rates. Further details of this model, together with a discussion of its limitations and domain of validity, are given in Refs. $[9,14]$.

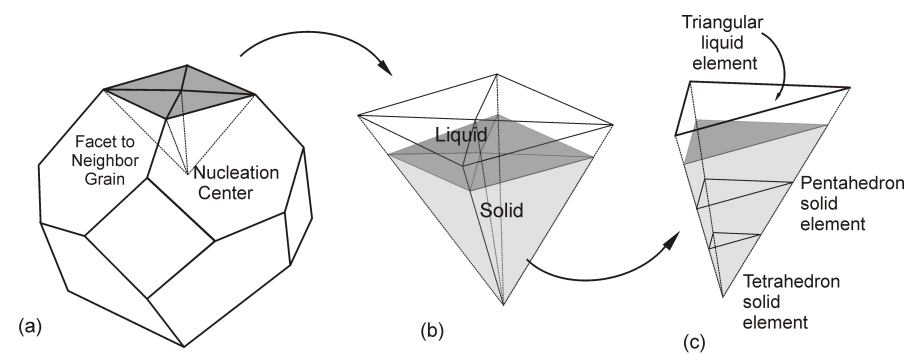

(c)
Figure 1. (a) a Voronoi region, or polyhedral final grain, (b) a small pyramidal subdivision showing both the solid portion (grey) and liquid portion (transparent), (c) a single tetrahedron decomposed into a set of solid elements

\subsection{Fluid flow Module}

The Navier-Stokes equations can be simplified if one considers the specific geometries of the interconnected liquid channels that remain between the solidifying polyhedral grains of the 
solidification module. In each triangular liquid prism separating two adjacent grain facets, it is assumed that the flow is parallel to the facet, i.e., the fluid velocity $\nu_{\ell}$ has only two components, $\nu_{x}$ and $\nu_{y}$, in a frame attached to the solid facet with the local $z^{\prime}$-axis perpendicular to it. Under such condition, the fluid velocity vector in between the polyhedral grains is given by [18]

$$
\overrightarrow{\nu_{\ell}}=\frac{1}{2 \mu_{\ell}} \vec{\nabla} p_{\ell}\left[z^{\prime 2}-h^{2}\right]
$$

where $\mathrm{h}$ is half-width of the liquid channel, $p_{\ell}$ the pressure, $\overrightarrow{\nu_{\ell}}$ the liquid velocity, $\mu_{\ell}$ the dynamic viscosity of the liquid. Combined with the conservation of mass, this equation gives [18]

$$
\frac{2 h^{3}}{3 \mu_{\ell}} \nabla^{2} p_{\ell}=2 \beta \nu^{*}+\left(v_{s n+}-v_{s n-}\right)+\frac{2 h}{K_{\ell}} \frac{\partial p_{\ell}}{\partial t}
$$

where $\left(v_{s n+}-v_{s n-}\right)$ is the difference between the normal $\left(z^{\prime}\right)$ components of the velocity of the two neighbor grains, $\nu^{*}$ the solidification velocity of the solid-liquid interface, $\beta=\left(\rho_{s} / \rho_{\ell}-1\right)$ the shrinkage factor, $\rho_{s}$, and $\rho_{\ell}$ the densities of the liquid and solid, respectively, $K_{\ell}$ the liquid bulk modulus. The left hand side of Eq. 2 is the variation of pressure required to compensate the volume change (right hand side) induced by solidification shrinkage and solid deformation (i.e., change of liquid channel width). This equation is solved using a Finite Element (FE) code written in $\mathrm{C}++$. Since the flow within an element is assumed to flow parallel to the facets only, each triangular liquid channel is discretized using 3-node 2D triangular elements. Once the individual element matrices have been developed, they are assembled into a master matrix for the whole set of interconnected triangular elements (mass conservation is automatically conserved along triple lines). This global stiffness matrix is solved with a Conjugate Gradient linear iterative method using a free open access $\mathrm{C}++$ template library known as IML $++[22]$.

\subsection{Semi-solid Deformation Module}

The geometry for simulating the semi-solid deformation consists of solid grains surrounded by liquid film channels. Within each grain, deformation is rather homogeneous and modeled using a continuum FE method, whilst within the entire domain, possibly discontinuous deformation is modeled using Discrete Element Method (DEM). Each solid tetrahedral element from the solidification calculation is split into three solid elements, a tetrahedron and two pentahedra, as shown in Fig. 1(c) for the FE calculation. As a result, there are approximately 120 solid elements per grain for the calculation. The output of the SM is automatically output as a finite element mesh using a $\mathrm{C}++$ subroutine within GMS-3D and the numerical simulation of the SDM is performed using the commercial code Abaqus 6.9TM. The mechanical behavior of these solid elements is assumed to be elasto-viscoplastic. The Young's modulus of the solid phase is assumed to be $E_{\text {solid }}=10 \mathrm{GPa}$, while the Poisson's ratio was taken as $\nu_{\text {solid }}=0.30$ [7]. The flow stress is based on the viscoplastic Ludwik's equation

$$
\sigma(\varepsilon, \dot{\varepsilon}, T)=k_{s}(T) \varepsilon^{n(T)} \dot{\varepsilon}^{m(T)}
$$

where $\sigma$ is the stress, $\varepsilon$ the total plastic strain experienced by the material, $\dot{\varepsilon}$ the strain rate, $k_{s}$ a flow stress coefficient, $n$ the strain hardening parameter and $m$ the strain rate sensitivity of the material. The values for these coefficients are as follows: $m=0.192, n=0$, and $k_{s}=40 M$ Pa.s [19]. In this simulation, it is assumed that the resistance of a liquid channel to separation is due only to the pressure that is exerted on the solid liquid interfaces. Furthermore, a hard contact pressure-overclosure relationship is used to prevent solid grain penetration. Shearing of the channel is modeled through a shearing parameter $E_{c}$, which is the only adjustable parameter of the model. This parameter is adjusted by comparing the mechanical response of the system to an imposed displacement with the semi-solid tensile tests available in the literature [7]. 


\subsection{Failure Module}

In order to model hot tearing, a criterion for this defect's initiation and propagation is necessary. Although multiple criteria have been suggested in the literature [see review by Eskin et al.[23]], the pressure drop due to shrinkage and deformation is a key parameter of most recentlydeveloped hot tearing criteria. In the present study, it is assumed that there is a contact between liquid and atmosphere, which is the case of semi-solid tensile tests used to investigate hot tearing (e.g.[3]). Accordingly, the criterion can be estimated from the overpressure required to overcome capillary forces at the liquid-atmosphere interface as illustrated in Fig. 2. The Young-Laplace equation of the penetrating void (assumed cylindrical in shape) is given by:

$$
p_{a}-p_{\ell}=\frac{\gamma}{R}=\frac{\gamma \cos \Theta}{h}
$$

where $p_{a}$ is the atmospheric pressure, $\Theta$ is the dihedral angle and $\gamma$ the surface tension at the void-liquid interface. Considering that a thin oxide skin can have formed between the void and the liquid, the value of $\gamma$ can be estimated as [24]

$$
\gamma=\sigma_{\ell o}+\sigma_{o g}+\tau \delta
$$

where $\sigma_{\ell o}$ and $\sigma_{o g}$ are the interfacial energy between oxide skin and liquid, oxide skin and air, respectively, and $\delta$ and $\tau$ are the thickness and the tensile strength of the oxide layer. The $\gamma \cos \Theta$ value has been fixed to $3.2 \mathrm{Jm}^{-2}$ in the present simulations [24]. Thus, the hot tear starts to propagate into a liquid channel connected to the atmosphere once $p_{\ell}$ is such that

$$
p_{\ell}=p_{a}-\frac{3.2}{h}
$$

Since the width of the widest channel $h_{\max }$ increases during a semi-solid tensile test, the maximal $p_{\ell}$, i.e. $p_{\ell, \max }^{c}=p_{a}-\frac{3.2}{h_{\max }}$ within the widest channel also increases during tensile deformation.

\subsection{Hydro-mechanical coupling}

Coupling in this hydro-mechanical model occurs by carrying out each of the FFM, SDM, and FM simulations incrementally at a given fraction solid (i.e. SM output), with iterations between each increment, until bulk fracture occurs. Assuming an initial value for liquid pressure $p_{\ell}=p_{\ell 0}$, the simulation begins with the SDM calculation. The mechanical response of the system to the imposed displacement $\Delta u=\dot{\varepsilon} L \Delta t$, where $\dot{\varepsilon}$ is the bulk strain rate and $\mathrm{L}$ the dimension of the RVE is calculated and then imported into the FFM. The new thickness and the value $\left(v_{s n+}-v_{s n-}\right)$ of all the channels is then calculated using the normal strain, $\varepsilon_{z^{\prime}}$ of each channel. With this information, the pressure $p_{\ell}$ is calculated using the FFM. The estimated $p_{\ell}$ for the SDM calculation is then modified for the next iteration based on the new $p_{\ell}$ found in each liquid channel network. Although the pressure gradient through a liquid channel network is the driving force for the fluid flow in this network, it is negligible for the SDM calculations due to the size of the RVE and thus it can be assumed that the liquid pressure is uniform. Next, the pressure in a channel is compared to failure criterion of the FM. When a hot tear forms in a channel, it is assumed that the liquid is sucked into the rest of the liquid network and the channel becomes dry. Hence when the FM predicts a hot tear has formed in a given channel, the pressure in this channel is set to $p_{a}$ for the SDM calculation. However, it does no longer participate to the overall feeding in the FFM calculation and the channel is closed by setting its displacement (local deformation) to $-2 \mathrm{~h}\left(\varepsilon_{z^{\prime}}=-1\right)$. The iterations are continued until the $p_{\ell}$ value converges, at which point the process is repeated for the next increment. 


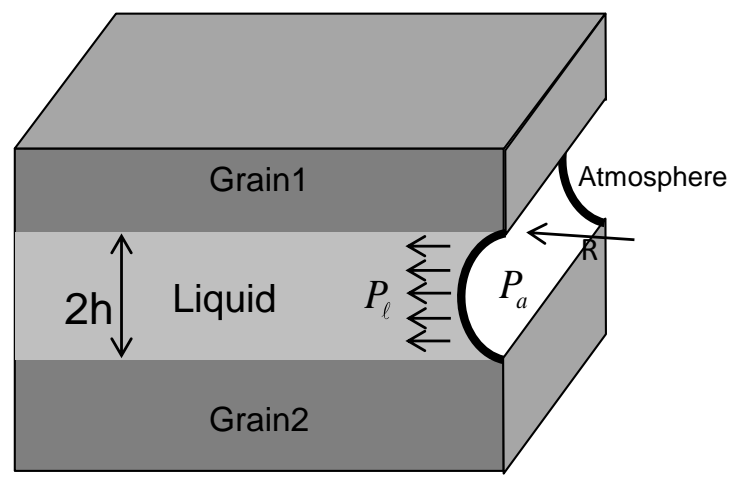

Figure 2. Schematic of two grains and a liquid channel within which a meniscus with a hemi-cylindrical shape starts forming.

\section{Results and Discussion}

All the results presented hereafter are shown by setting $p_{a}$ to zero. In order to validate the predictions of the FFM, the permeability $\mathcal{K}$ of the mushy zone is calculated and compared to the value predicted by Carman-Kozeny's relationship [25]:

$$
\mathcal{K} S_{s}^{2}=\frac{\left(1-g_{s}\right)^{3}}{5}
$$

where $\mathcal{K}$ is normalized by the square of the specific solid-liquid interfacial area $S_{s}^{2}$, and $g_{s}$ is the volume fraction of solid. For the calculation, an isothermal RVE of the mushy zone with various $g_{s}$ is considered with the following boundary conditions: a constant pressure $p_{0}$ on the top surface, a fixed flux $q$ on the bottom surface while the four lateral surfaces are closed. Darcy's law is then used to calculate the permeability:

$$
\mathcal{K}=\frac{q \mu_{\ell}}{\frac{p_{1}-p_{0}}{L}}
$$

where $p_{1}$ is the average pressure found on the bottom side of the volume element. Figure 3 shows the pressure contours for three values of $g_{s}\left(g_{s}=0.60,0.90\right.$ and 0.98$)$ where $q=100 \mu \mathrm{m}$ $\mathrm{s}^{-1}$ and $p_{0}=0$. In this figure, although the pressure is defined only in the liquid channels, the gray color is also represented within the grains for visibility. The calculated liquid flow in each channel is represented with a plate of finite thickness proportional to the local flow normalized by the overall flow. The fluid flow is fairly well distributed in the liquid channels for the low $g_{s}$ case (Fig.3(a)). However, localized feeding occurs along a few preferential paths at $g_{s}=0.90$ (Fig. 3(b)) and even more at $g_{s}=0.98$ (Fig. 3(c)). Also, note that the maximum local pressure drop $\left(p_{0}-p_{1}\right)$ increases with $g_{s}$; the maximum local pressure drop for $g_{s}=0.98$ is approximately 4 orders of magnitude larger than the maximum local pressure drop for $g_{s}=0.60$.

Figure 4 shows a comparison of the variation in normalised permeability, $\mathcal{K} S_{s}^{2}$, with $g_{s}$ as calculated using Carman-Kozeny relation (Eq. 7) and the present FFM. The two curves are in excellent agreement, especially for $g_{s}>0.4$. Please note that at low $g_{s}$, the liquid channels are wide and thus the Poiseuille laminar flow assumption is no longer valid, while the flow contribution at vortices, i.e., points where 4 grains meet, cannot be neglected anymore.

Figure 5 shows the simulated stress-strain predictions at $g_{s}=0.96$ and $g_{s}=0.94$ at a strain rate of $\dot{\varepsilon}=10^{-3} \mathrm{~s}^{-1}$. The $E_{c}$ value in these simulations is fixed to $2.6 \mathrm{MPa}$ and all the surfaces are assumed to be closed for the FFM calculation. For the SDM calculation a symmetry boundary condition has been applied to the surfaces $\mathrm{x}=0, \mathrm{y}=0$ and $\mathrm{z}=0$ of the RVE. The surface $x=L_{x}$ is connected to a reference node which is displaced at a fixed velocity in x-direction, while the surfaces $y=L_{y}$ and $z=L_{z}$ are free to move. The variables $L_{x}, L_{y}$, and $L_{z}$ refer to the dimensions of the domain in the $\mathrm{x}, \mathrm{y}$, and $\mathrm{z}$ directions, respectively. Locations 

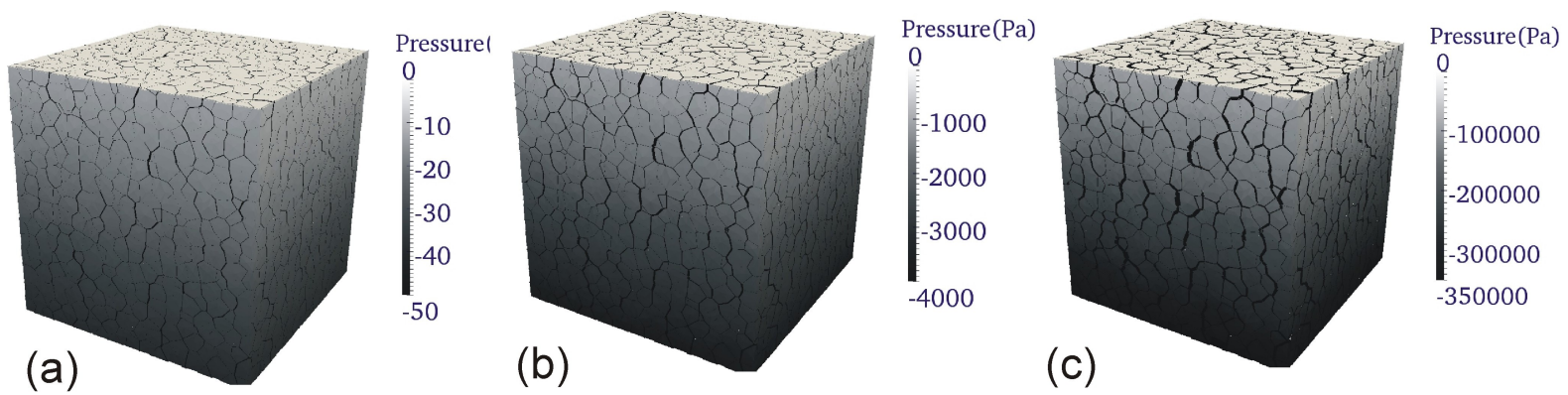

Figure 3. Fluid flow through a mushy zone volume element $\left(2 \times 2 \times 2 \mathrm{~mm}^{3}\right)$ consisting of 8000 grains for (a) $g_{s}=0.6$ (b) $g_{s}=0.90$, (c) $g_{s}=0.98$; the line width used to show each channel is proportional to the local flow normalized by the total flow within the volume element

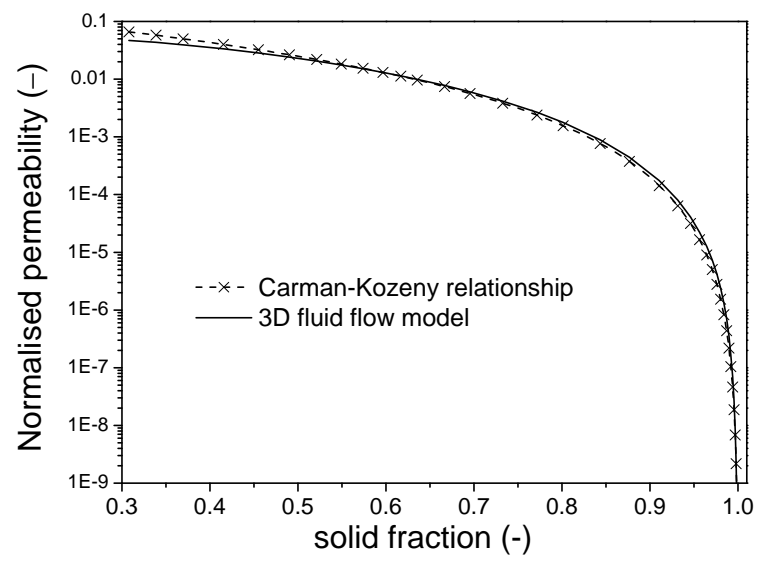

Figure 4. Normalized permeability, $\mathcal{K} S_{s}^{2}$, with $g_{s}$ for a random network of grains calculated using the 3D fluid flow model and CarmanKozeny's relationship

(a), (b), and (c) will be discussed below. As can be seen, the stress increases with increasing strain, as expected, and reaches a maximum value $\sigma_{\max }$ before overall failure occurs. In Figure 6 , the intergranular liquid pressure drop that occurs during the tensile deformation is reported (continuous curves, output of the FFM), along with the failure pressure $p_{\ell, \max }^{c}$ associated with the widest channel (dashed curves, output of the FM). The two curves $p_{\ell}$ and the $p_{\ell, \max }^{c}$ cross at the strains $\varepsilon \cong 0.009$ and $\varepsilon \cong 0.012$ for $g_{s}=0.96$ and $g_{s}=0.94$, respectively. At this point, which corresponds to a strain one increment beyond location (a) for the curve $g_{s}=0.96$, the first crack has now initiated in the widest channel connected to ambient air. As can be seen by comparing this portion of the curves in Figs. 5 and 6, the overall stress within the RVE continues to increase, but at a lower rate. With increasing strain, the crack continues to propagate within the RVE, and the rate $d \sigma / d \varepsilon$ continues to decrease until $\sigma_{\max }$ near (c) is reached, and the rate becomes negative.

Figure 7 shows the contour plots of the Von Mises stress in $(\mathrm{MPa})$ for $g_{s}=0.94$ at the three strains corresponding to (a), (b), and (c) of Figs.5 and 6. In this figure, the channels in which the Von Mises stress is not represented (white channels) correspond to those where a hot tear has formed. Finally, in Fig. 8, a comparison is made between the simulated maximum stress (stress at fracture) and the experimental results for the same $\mathrm{Al}-2 \mathrm{wt} . \% \mathrm{Cu}$ alloy from [7], over a range of fractions of solid between 0.8 and 0.99 at a strain rate of $10^{-3} \mathrm{~s}^{-1}$. As can be seen, this model correctly reproduces the general trends of the experimental results. However, for $g_{s}<0.94$, it seems that this model overestimates the strength of this alloy. 


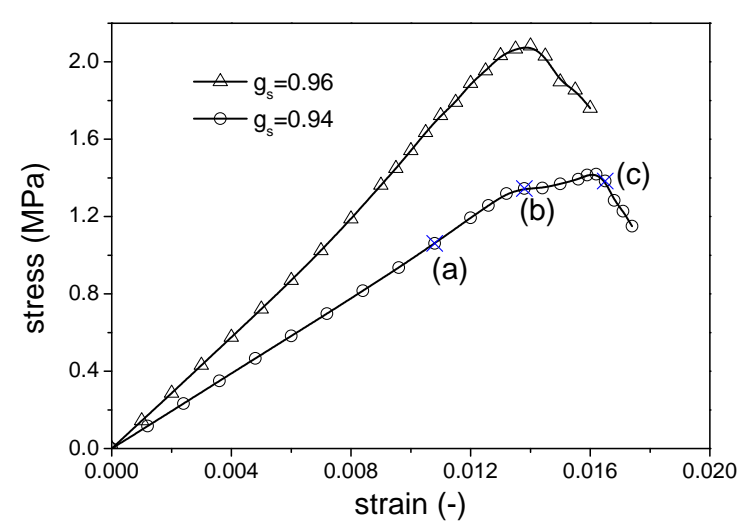

Figure 5. Calculated tensile behavior of partially solidified $\mathrm{Al}-\mathrm{Cu}$ alloys $\mathrm{Al}-2 \mathrm{wt} . \% \mathrm{Cu}$ : stress vs. strain curves for $\triangle g_{s}=0.96$ and $g_{s}=0.94$.

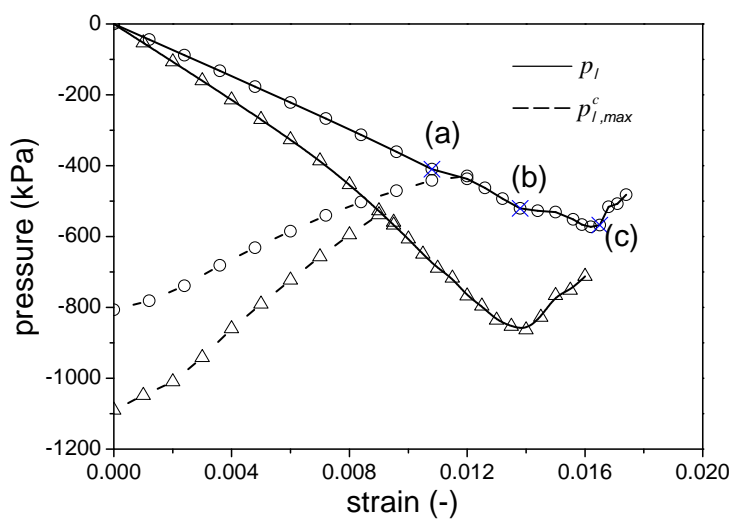

Figure 6. Liquid pressure drop (continues curves) and maximum value of the cavitation pressure (dashed curves) in the mushy zone as a function of strain corresponding to Fig.5
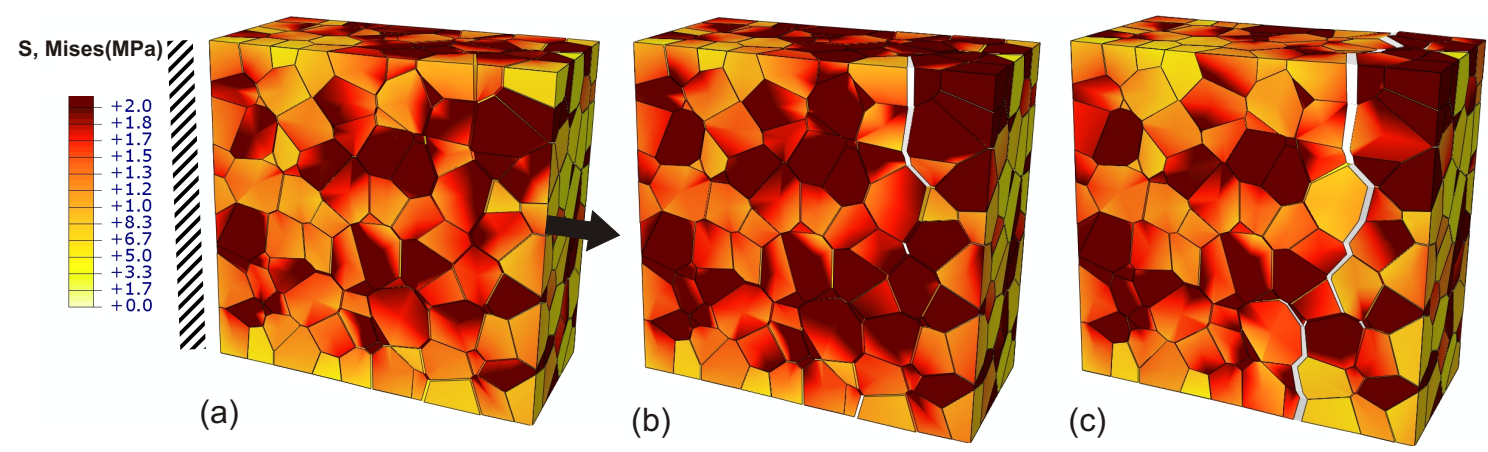

Figure 7. Contour plots of the Von Mises stress at various deformation stages and for $g_{s}=0.94$. The white channels correspond to those where a hot tear has formed.

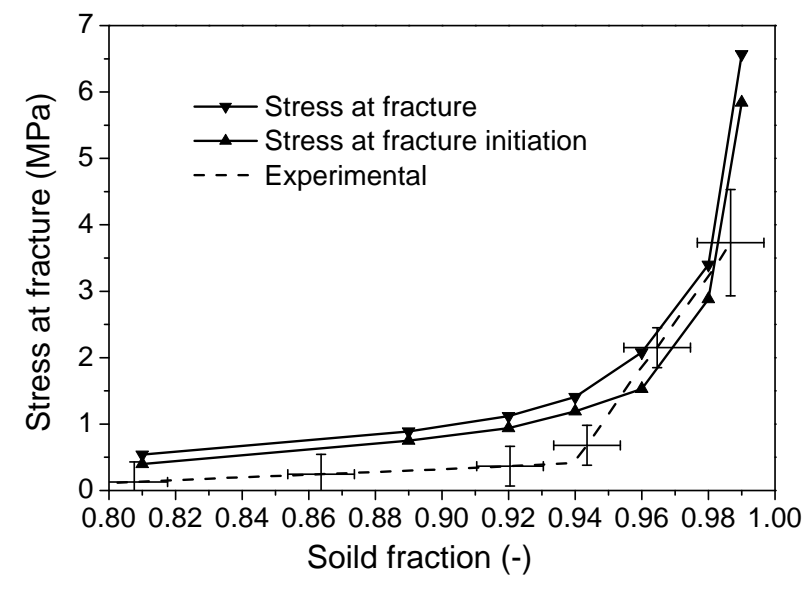

Figure 8. Comparison between the tensile strength measured in semi-solid Al2 wt.\% $\mathrm{Cu}$ alloy $[7]$ (dashed line curve) and simulation results (continuous curves).

\section{Conclusion}

A 3D granular hydro-mechanical coupled model has been developed to predict hot tearing formation in solidifying alloys. This model is made up of four separate 3D modules; (I) a 
solidification module (II) a semi-solid deformation module (III) a fluid flow module and (IV) a failure module. This model is able to predict the overall response of the semi-solid alloys to an externally applied strain before and after fracture initiation, while accounting for the localization of strains at grain boundaries. The strength of semi-solid alloys at various $g_{s}$ predicted with this model agree well with the experimental results.

\section{Acknowledgements}

The authors would like to thank the Swiss Competence Centre for Materials Science and Technology (CCMX), and partner companies within the thematic area "Multi-scale, multiphenomena modelling of metallic systems" for funding this research.

\section{References}

[1] Gourlay C M, Dahle A K, Nagira T, Nakatsuka N, Nogita K, Uesugi K and Yasuda H 2011 Acta Materialia 59 4933-4943

[2] Gourlay C M and Dahle A K 2007 Nature 445 70-73

[3] Terzi S, Salvo L, Sury M, Limodin N, Adrien J, Maire E, Pannier Y, Bornert M, Bernard D, Felberbaum M, Rappaz M and Boller E 2009 Scripta Materialia 61449 - 452

[4] Bernard D, Nielsen O, Salvo L and Cloetens P 2005 Materials Science and Engineering a-Structural Materials Properties Microstructure and Processing 392 112-120

[5] Monroe C and Beckermann C 2005 Materials Science and Engineering A 413-414 30-36

[6] M'Hamdi M, Mo A and Martin C 2002 Metallurgical and materials transactions a 33 2081-2093

[7] Ludwig O, Drezet J M, Martin C and Sury M 2005 Metallurgical and Materials Transactions A 36 1525-1535

[8] Vernède S and Rappaz M 2006 Philosophical Magazine 86 3779-3794

[9] Phillion A B, Desbiolles J L and Rappaz M 2009 Modeling Of Casting, Welding, And Advanced Solidification Processes - XII (Minerals, Metals \& Materials Soc, 184 Thorn Hill Rd, Warrendale, Pa 15086-7514 Usa) pp 353-360

[10] Mathier V, Jacot A and Rappaz M 2004 Modelling and Simulation in Materials Science and Engineering 12 479-490

[11] Phillion A B, Cockcroft S L and Lee P D 2008 Acta Materialia 56 4328-4338

[12] Vernède S, Dantzig J A and Rappaz M 2009 Acta Materialia 57 1554-1569

[13] Lahaie D J and Bouchard M 2001 Metallurgical and Materials Transactions B 32 697-705

[14] Sistaninia M, Phillion A, Drezet J M and Rappaz M 2011 Metallurgical and Materials Transactions A 42 239-248

[15] Phillion A, Vernede S, Rappaz M, Cockcroft S and Lee P D 2009 International Journal of Cast Metals Research 22 240-243

[16] Vernède S, Jarry P and Rappaz M 2006 Acta Materialia 544023 - 4034

[17] Dijkstra W O, Vuik C, Dammers A J and Katgerman L 2004 ed Rappaz M, Beckermann C and Trivedi R (TMS) pp 151-156

[18] Sistaninia M, Phillion A B, Drezet J M and Rappaz M 2011 Acta Materialia (under review)

[19] Fuloria D and Lee P 2009 Acta Materialia 57 5554-5562

[20] Magnin B, Maenner L, Katgerman L and Engler S 1996 Materials Science Forum 217-222 1209-1214

[21] Vernède S and Rappaz M 2007 Acta Materialia 551703 - 1710

[22] Dongarra J, Lumsdaine R, Pozo R and Remington K 1994 Proceedings of the second object oriented numerics conference pp 214-218

[23] Eskin D G, Suyitno and Katgerman L 2004 Progress in Materials Science 49 629-711

[24] Syvertsen M 2006 Metallurgical and Materials Transactions B: Process Metallurgy and Materials Processing Science 37 495-504

[25] Carman P 1937 Transactions of the Institution of Chemical Engineers 15 150-166 